\title{
APPROXIMATION OF FRACTIONAL POSITIVE STABLE CONTINUOUS-TIME LINEAR SYSTEMS BY FRACTIONAL POSITIVE STABLE DISCRETE-TIME SYSTEMS
}

\author{
TADEUSZ KACZOREK
}

Faculty of Electrical Engineering

Białystok Technical University, ul. Wiejska 45D, 15-351 Białystok, Poland

e-mail:kaczorek@isep.pw.edu.pl

\begin{abstract}
Fractional positive asymptotically stable continuous-time linear systems are approximated by fractional positive asymptotically stable discrete-time systems using a linear Padé-type approximation. It is shown that the approximation preserves the positivity and asymptotic stability of the systems. An optional system approximation is also discussed.
\end{abstract}

Keywords: Padé approximation, fractional system, linear positive system.

\section{Introduction}

In positive systems inputs, state variables and outputs take only non-negative values. Examples of positive systems are industrial processes involving chemical reactors, heat exchangers and distillation columns, storage systems, compartmental systems, or water and atmospheric pollution models. A variety of models having positive linear behavior can be found in engineering, management science, economics, social sciences, biology and medicine, etc. Positive linear systems are defined on cones and not on linear spaces. Therefore, the theory of positive systems is more complicated and less advanced. An overview of state of the art in positive systems theory is given by Farina and Rinaldi (2000) as well as Kaczorek (2002).

The stability of positive linear systems was investigated by Farina and Rinaldi (2000) as well as Kaczorek (2002), and that of fractional linear systems by Busłowicz (2008; 2012), Busłowicz and Kaczorek (2009), as well as Kaczorek (2011). The problem of preservation of positivity by approximating the continuous-time linear systems by the corresponding discrete-time linear systems was addressed by Kaczorek (1999).

A linear Padé-type approximation of the exponential matrix of positive asymptotically stable continuous-time linear systems was applied by Kaczorek (2013). It was shown that the approximation preserves the positivity and asymptotic stability of the systems.

In this paper the linear Padé-type approximation will be applied to fractional positive continuous-time linear systems.

The paper is organized as follows. In Section 2, basic definitions and theorems concerning positive standard and fractional continuous-time and discrete-time linear systems are recalled. In Section 3, the main result of the paper is presented. It is shown that the approximation preserves the positivity and asymptotic stability of the systems. Concluding remarks are given in Section 4.

The following notation will be used:

- $\mathbb{R}$ : the set of real numbers,

- $\mathbb{R}^{n \times m}$ : the set of $n \times m$ real matrices,

- $\mathbb{R}_{+}^{n \times m}$ : the set of $n \times m$ matrices with nonnegative entries and $\mathbb{R}_{+}^{n}=\mathbb{R}_{+}^{n \times 1}$,

- $M_{n}$ : the set of $n \times n$ Metzler matrices (real matrices with nonnegative off-diagonal entries),

- $M_{n s}$ : the set of $n \times n$ asymptotically stable Metzler matrices,

- $I_{n}$ : the $n \times n$ identity matrix.

\section{Preliminaries and problem formulation}

2.1. Standard positive systems. Consider the continuous-time linear system

$$
\dot{x}(t)=A_{c} x(t)+B_{c} u(t), \quad x(0)=x_{0},
$$


where $x(t) \in \mathbb{R}^{n}, u(t) \in \mathbb{R}^{m}$ are the state and input vectors and $A_{c} \in \mathbb{R}^{n \times n}, B_{c} \in \mathbb{R}^{n \times m}$.

Definition 1. (Farina and Rinaldi, 2000; Kaczorek, 2002) The continuous-time system (1) is called (internally) positive if $x(t) \in \mathbb{R}_{+}^{n}, t \geq 0$ for any initial conditions $x(0)=x_{0} \in \mathbb{R}_{+}^{n}$ and all inputs $u(t) \in \mathbb{R}_{+}^{m}, t \geq 0$.

Theorem 1. (Farina and Rinaldi, 2000; Kaczorek, 2002) The continuous-time system (1) is positive if and only if

$$
A_{c} \in M_{n}, \quad B_{c} \in \mathbb{R}_{+}^{n \times m} .
$$

Definition 2. (Farina and Rinaldi, 2000; Kaczorek, 2002) The positive continuous-time system (1) is called asymptotically stable if for $u(t)=0, t \geq 0$,

$$
\lim _{t \rightarrow \infty} x(t)=0 \quad \text { for all } \quad x_{0} \in \mathbb{R}_{+}^{n} .
$$

Theorem 2. (Farina and Rinaldi, 2000; Kaczorek, 2002) The positive continuous-time system (1) is asymptotically stable if and only if all coefficients of the polynomial

$$
\operatorname{det}\left[I_{n} s-A_{c}\right]=s^{n}+a_{n-1} s^{n-1}+\cdots+a_{1} s+a_{0}
$$

are positive, i.e., $a_{i}>0$ for $i=0,1, \ldots, n-1$.

Now let us consider the discrete-time linear system

$$
x_{i+1}=A_{d} x_{i}+B_{d} u_{i}, \quad i \in \mathbb{Z}_{+},
$$

where $x_{i} \in \mathbb{R}^{n}, u_{i} \in \mathbb{R}^{m}$ are the state and input vectors and $A_{d} \in \mathbb{R}^{n \times n}, B_{d} \in \mathbb{R}^{n \times m}$.

Definition 3. (Farina and Rinaldi, 2000; Kaczorek, 2002) The discrete-time system (5) is called (internally) positive if $x_{i} \in \mathbb{R}_{+}^{n}, i \in Z_{+}$, for any initial conditions $x_{0} \in \mathbb{R}_{+}^{n}$ and all inputs $u_{i} \in \mathbb{R}_{+}^{m}, i \in \mathbb{Z}_{+}$.

Theorem 3. (Farina and Rinaldi, 2000; Kaczorek, 2002) The discrete-time system (5) is positive if and only if

$$
A_{d} \in \mathbb{R}_{+}^{n \times n}, \quad B_{d} \in \mathbb{R}_{+}^{n \times m} .
$$

Definition 4. (Farina and Rinaldi, 2000; Kaczorek, 2002) The positive discrete-time system (5) is called asymptotically stable if for $u_{i}=0, i \in \mathbb{Z}_{+}$,

$$
\lim _{i \rightarrow \infty} x_{i}=0 \text { for all } x_{0} \in \mathbb{R}_{+}^{n} .
$$

Theorem 4. (Farina and Rinaldi, 2000; Kaczorek, 2002) The positive discrete-time system (5) is asymptotically stable if and only if all coefficients of the polynomial

$$
\begin{aligned}
& \operatorname{det}\left[I_{n}(z+1)-A_{d}\right] \\
& \quad=z^{n}+\bar{a}_{n-1} z^{n-1}+\cdots+\bar{a}_{1} z+\bar{a}_{0}
\end{aligned}
$$

are positive, i.e., $\bar{a}_{i}>0 \quad$ for $\quad i=0,1, \ldots, n-1$.
It is well-known that if sampling is applied to the continuous-time system (1) then the corresponding discrete-time system (5) has the matrices

$$
A_{d}=e^{A_{c} h}, \quad B_{d}=\int_{0}^{h} e^{A_{c} t} B_{c} \mathrm{~d} t
$$

where $h>0$ is the sampling period.

If $\operatorname{det} A_{c} \neq 0$ and rank $B_{c}=m$, then from (9) we have

$$
B_{d}=A_{c}^{-1}\left(e^{A_{c} h}-I_{n}\right) B_{c}
$$

and

$$
\operatorname{rank} B_{d}=m
$$

since $\operatorname{det}\left[e^{A_{c} h}-I_{n}\right] \neq 0$.

2.2. Fractional positive systems. Consider the continuous-time fractional linear system described by the state equation

$$
\frac{\mathrm{d}^{\alpha} x(t)}{\mathrm{d} t^{\alpha}}=A_{c} x(t)+B_{c} u(t), \quad 0<\alpha \leq 1,
$$

where $x(t) \in \mathbb{R}^{n}, u(t) \in \mathbb{R}^{m}$ are respectively the state and input vectors, and $A_{c} \in \mathbb{R}^{n \times n}, B_{c} \in \mathbb{R}^{n \times m}$,

$$
\begin{aligned}
\frac{\mathrm{d}^{\alpha} x(t)}{\mathrm{d} t^{\alpha}} & ={ }_{0} D_{t}^{\alpha} x(t)=\frac{1}{\Gamma(1-\alpha)} \int_{0}^{t} \frac{\dot{x}(\tau)}{(t-\tau)^{\alpha}} \mathrm{d} \tau, \\
\dot{x}(\tau) & =\frac{\mathrm{d} x(\tau)}{\mathrm{d} \tau}
\end{aligned}
$$

is the Caputo fractional derivative, while

$$
\Gamma(x)=\int_{0}^{\infty} e^{-t} t^{x-1} \mathrm{~d} t, \quad \operatorname{Re}(x)>0 .
$$

is the Euler gamma function.

Theorem 5. (Kaczorek, 2011) The solution of Eqn. (12) has the form

$$
\begin{aligned}
& x(t)=\Phi_{0}(t) x_{0}+\int_{0}^{t} \Phi(t-\tau) B_{c} u(\tau) \mathrm{d} \tau, \\
& x(0)=x_{0}
\end{aligned}
$$

where

$$
\Phi_{0}(t)=\sum_{k=0}^{\infty} \frac{A_{c}^{k} t^{k \alpha}}{\Gamma(k \alpha+1)}, \quad \Phi(t)=\sum_{k=0}^{\infty} \frac{A_{c}^{k} t^{(k+1) \alpha-1}}{\Gamma[(k+1) \alpha]} .
$$

Definition 5. The fractional continuous-time system (12) is called (internally) positive fractional if the state vector $x(t) \in \mathbb{R}_{+}^{n}, t \geq 0$, for all initial conditions $x_{0} \in \mathbb{R}_{+}^{n}$ and all inputs $u(t) \in \mathbb{R}_{+}^{m}, t \geq 0$.

Theorem 6. (Kaczorek, 2011) The fractional continuoustime system (12) is internally positive if and only if

$$
A_{c} \in M_{n}, \quad B_{c} \in \mathbb{R}_{+}^{n \times m} .
$$


Theorem 7. (Kaczorek, 2011) The fractional positive continuous-time system (12) is asymptotically stable if and only if the eigenvalues of $A_{c}$ are located in the open left half of the complex plane.

Theorem 8. (Farina and Rinaldi, 2000; Kaczorek, 2002; 2011) The fractional positive continuous-time system (12) is asymptotically stable if and only if all coefficients of the polynomial (4) are positive, i.e., $a_{i}>0$ for $i=$ $0,1, \ldots, n-1$.

Now let us consider the fractional discrete-time linear system

$$
\Delta^{\alpha} x_{i+1}=A_{d} x_{i}+B_{d} u_{i}, \quad i \in \mathbb{Z}_{+}, \quad 0<\alpha<1,
$$

where $x_{i} \in \mathbb{R}^{n}$ is the state vector, $u_{i} \in \mathbb{R}^{m}$ is the input vector and $A_{d} \in \mathbb{R}^{n \times n}, B_{d} \in \mathbb{R}^{n \times m}$ while

$$
\begin{aligned}
& \Delta^{\alpha} x_{i}=x_{i}+\sum_{j=1}^{i}(-1)^{j}\left(\begin{array}{c}
\alpha \\
j
\end{array}\right) x_{i-j},\left(\begin{array}{c}
\alpha \\
j
\end{array}\right) \\
& =\left\{\begin{array}{cc}
1 & \text { for } j=0, \\
\frac{\alpha(\alpha-1) \ldots(\alpha-j+1)}{j !} & \text { for } j=1,2, \ldots
\end{array}\right.
\end{aligned}
$$

is the fractional $\alpha$ order difference of $x_{i}$.

Substituting (19) into (18) we obtain

$$
\begin{aligned}
x_{i+1}= & A_{\alpha} x_{i}+\sum_{j=2}^{i+1}(-1)^{j+1}\left(\begin{array}{c}
\alpha \\
j
\end{array}\right) x_{i-j+1} \\
& +B_{d} u_{i}, \quad i \in \mathbb{Z}_{+},
\end{aligned}
$$

where

$$
A_{\alpha}=A_{d}+I_{n} \alpha .
$$

Theorem 9. The solution of Eqn. (20) has the form

$$
x_{i}=\Phi_{i} x_{0}+\sum_{j=0}^{i-1} \Phi_{i-j-1} B_{d} u_{i},
$$

where the matrix $\Phi_{i}$ can be computed from the formula

$$
\begin{aligned}
\Phi_{i+1} & =\Phi_{i} A_{\alpha}+\sum_{j=2}^{i+1}(-1)^{j+1}\left(\begin{array}{c}
\alpha \\
j
\end{array}\right) \Phi_{i-j+1}, \\
\Phi_{0} & =I_{n} .
\end{aligned}
$$

Definition 6. (Kaczorek, 2011) The fractional discrete-time system (18) is called (internally) positive if $x_{i} \in \mathbb{R}_{+}^{n}, i \in \mathbb{Z}_{+}$, for all initial conditions $x_{0} \in \mathbb{R}_{+}^{n}$ and all input sequences $u_{i} \in \mathbb{R}_{+}^{m}, i \in \mathbb{Z}_{+}$.

Theorem 10. (Farina and Rinaldi, 2000; Kaczorek, 2002; 2011) The fractional discrete-time system (18) is positive if and only if

$$
A_{\alpha} \in \mathbb{R}_{+}^{n \times n}, \quad B_{d} \in \mathbb{R}_{+}^{n \times m} .
$$

Theorem 11. (Kaczorek, 2011) The fractional positive discrete-time system (18) is asymptotically stable if and only if the positive system

$$
x_{i+1}=\left(A_{d}+I_{n}\right) x_{i}
$$

is asymptotically stable.

Theorem 12. (Kaczorek, 2011) The fractional positive discrete-time system (18) is asymptotically stable if and only if all coefficients of the polynomial

$$
\operatorname{det}\left[I_{n} z-A_{d}\right]=z^{n}+\hat{a}_{n-1} z^{n-1}+\cdots+\hat{a}_{1} z+\hat{a}_{0}
$$

are positive, i.e., $\hat{a}_{i}>0$ for $i=0,1, \ldots, n-1$.

\section{Application of a linear Padé approximation}

In a similar way as for standard linear systems (Kaczorek, 2013), it can be easily shown that if sampling is applied to the fractional continuous-time system (12) then the corresponding fractional discrete-time system (20) has the matrices

$$
\begin{aligned}
& A_{\alpha}=\Phi_{0}(h), \\
& B_{d}=\int_{0}^{h} \Phi(t) B_{c} \mathrm{~d} t,
\end{aligned}
$$

where $\Phi_{0}(t)$ and $\Phi(t)$ are defined by (16).

In this paper the matrix $A_{d}$ will be approximated by

$$
A_{d}=\left[A_{c}+I_{n} \beta\right]\left[I_{n} \beta-A_{c}\right]^{-1},
$$

where the coefficient $\beta>0$ is chosen so that $A_{c}+I_{n} \beta \in$ $\mathbb{R}$ and $\left[I_{n} \beta-A_{c}\right]^{-1} \in \mathbb{R}_{n}^{n \times n}, h>0$ is the sampling period. If $A_{c} \in M_{n s}$, then $\operatorname{det}\left[I_{n} \beta-A_{c}\right] \in \mathbb{R}_{=}^{n \times n}$ for any $\beta>0$.

For $\operatorname{det} A_{c} \neq 0$, using the Padé approximation from (27b) we obtain

$$
B_{d}=A_{c}^{-1}\left\{\left[A_{c}+I_{n} \beta\right]\left[I_{n} \beta-A_{c}\right]^{-1}-I_{n}\right\} B_{c} .
$$

Remark 1. Knowing $A_{c}, B_{c}$ and $h$ and using (27b) we may compute the exact matrix $B_{d}$.

Therefore, the fractional continuous-time linear system (12) can be approximated by the fractional discrete-time linear system (20) with the matrices $A_{d}$ and $B_{d}$ defined by (27b) and (28), respectively.

Theorem 13. If the fractional continuous-time system (12) is positive and asymptotically stable, then the corresponding fractional discrete-time system (20) with (27a) and (28b) is also positive and its matrix eigenvalues are located inside the unit circle $\left|z_{k}\right|<1$ for $k=1, \ldots, n$, and any sampling period $h>0$. 
Proof. If the fractional continuous-time system (12) is positive and asymptotically stable, then $A_{c} \in M_{n s}$, and for any $\beta \geq \max \left(-a_{i, i}^{c}\right)$ (where $a_{i, i}^{c}$ is the $i$-th diagonal entry of $\left.A_{c}, i=1,2, \ldots, n\right)$ we have $\left[A_{c}+I_{n} \beta\right] \in \mathbb{R}_{+}^{n \times n}$, $\left[I_{n} \beta-A_{c}\right]^{-1} \in \mathbb{R}_{+}^{n \times n}$ (Berman and Plemmons, 1994; Kaczorek, 2011) and $A_{d} \in \mathbb{R}_{+}^{n \times n}$. From (27b) (and also from (28)) we have $B_{d} \in \mathbb{R}_{+}^{n \times m}$ since $\Phi(t) \in \mathbb{R}_{+}^{n \times n}$, $t \geq 0$ and $B_{c} \in \mathbb{R}_{+}^{n \times m}$. Therefore, the corresponding fractional discrete-time system (20) is positive for any $h>0$ if the fractional continuous-time system (12) is positive and asymptotically stable since (Kaczorek, 2011)

$$
(-1)^{j+1}\left(\begin{array}{c}
\alpha \\
j
\end{array}\right)>0
$$

for $0<\alpha<1$ and $j=2,3, \ldots$.

If the fractional positive system 12 is asymptotically stable, then the real parts $\alpha_{k}$ of the eigenvalues $s_{k}=-\alpha_{k} \pm j \beta_{k}, k=1,2, \ldots, n$, of the matrix $A_{c}$ are negative. By Lemma A1 (see Appendix) and Theorem 11, from (A2) we obtain

$$
\begin{aligned}
\left|z_{k}\right| & =\left|\frac{\beta-\alpha_{k} \pm j \beta_{k}}{\beta+\alpha_{k} \mp j \beta_{k}}\right| \\
& =\frac{\sqrt{\left(\beta-\alpha_{k}\right)^{2}+\beta_{k}^{2}}}{\sqrt{\left(\beta+\alpha_{k}\right)^{2}+\beta_{k}^{2}}}<1 .
\end{aligned}
$$

Therefore, the eigenvalues of the matrix of the fractional positive discrete-time system (20) with (27a) and (28) are located inside the unit circle for any sampling period $h>$ 0 .

Remark 2. Note that the precision of the approximation of the fractional positive continuous-time system (12) by the fractional positive discrete-time system (20) depends on the choice of the coefficient $\beta$. It is recommended to choose the coefficient $\beta$ so that the square of the difference between the solutions of the continuous-time and discrete-time systems be minimal.

Example 1. Consider the fractional positive and asymptotically stable continuous-time linear system (12) with $0<\alpha<1$ and the matrices

$$
A_{c}=\left[\begin{array}{cc}
-2 & 1 \\
0 & -3
\end{array}\right], \quad B_{c}=\left[\begin{array}{l}
0 \\
1
\end{array}\right]
$$

The step response of the fractional continuous-time system with matrices (31) and zero initial conditions is given in Fig. 1.

Using (27a) and (28), we may compute the matrices $A_{d}$ and $B_{d}$ of the fractional discrete-time system (20) for

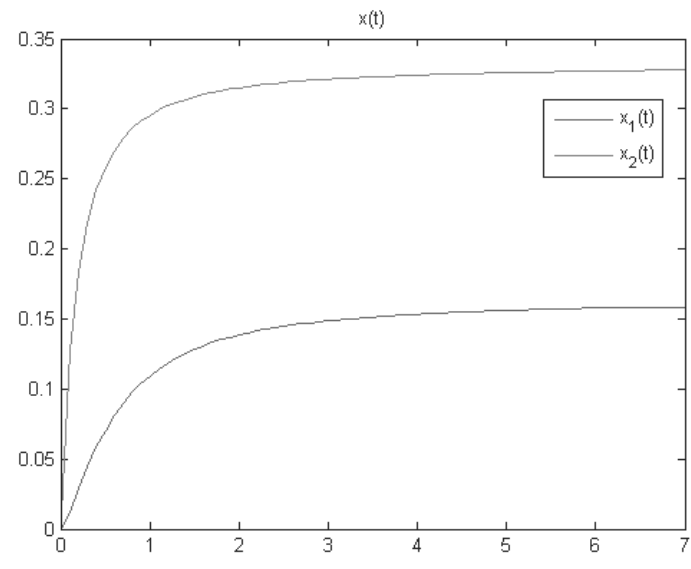

Fig. 1. Step response of the fractional continuous-time system (31).

$\beta=4$,

$$
\begin{aligned}
A_{d} & =\left[A_{c}+I_{n} \beta\right]\left[I_{n} \beta-A_{c}\right]^{-1} \\
& =\left[\begin{array}{ll}
2 & 1 \\
0 & 1
\end{array}\right]\left[\begin{array}{cc}
6 & -1 \\
0 & 7
\end{array}\right]^{-1} \\
& =\frac{1}{21}\left[\begin{array}{ll}
7 & 4 \\
0 & 3
\end{array}\right], \\
B_{d} & =\Phi\left(A_{c} h\right)=\left[\begin{array}{l}
0.0875267 \\
0.2365546
\end{array}\right],
\end{aligned}
$$

and

$$
\begin{aligned}
B_{d}= & A_{c}^{-1}\left\{\left[A_{c}+I_{n} \beta\right]\left[I_{n} \beta-A_{c}\right]^{-1}-I_{n}\right\} B_{c} \\
= & {\left[\begin{array}{cc}
-2 & 1 \\
0 & -3
\end{array}\right]^{-1}\left\{\frac{1}{21}\left[\begin{array}{ll}
7 & 4 \\
0 & 3
\end{array}\right]\right.} \\
& \left.-\left[\begin{array}{ll}
1 & 0 \\
0 & 1
\end{array}\right]\right\}\left[\begin{array}{l}
0 \\
1
\end{array}\right] \\
= & \frac{1}{21}\left[\begin{array}{l}
1 \\
6
\end{array}\right] .
\end{aligned}
$$

By Theorem 10, the fractional discrete-time system with (32) is positive.

The step response of the fractional discrete-time system with matrices (32) and zero initial conditions is given in Fig. 2.

The matrix $A_{c}$ given by (31) of fractional positive asymptotically stable continuous-time systems has the eigenvalues $s_{1}=-2, s_{2}=-3$. Using (A2) we obtain, for $\beta=4$,

$$
\begin{aligned}
& z_{1}=\frac{\beta+s_{1}}{\beta-s_{1}}=\frac{4-2}{4+2}=\frac{1}{3}, \\
& z_{2}=\frac{\beta+s_{2}}{\beta-s_{2}}=\frac{4-3}{4+3}=\frac{1}{7} .
\end{aligned}
$$




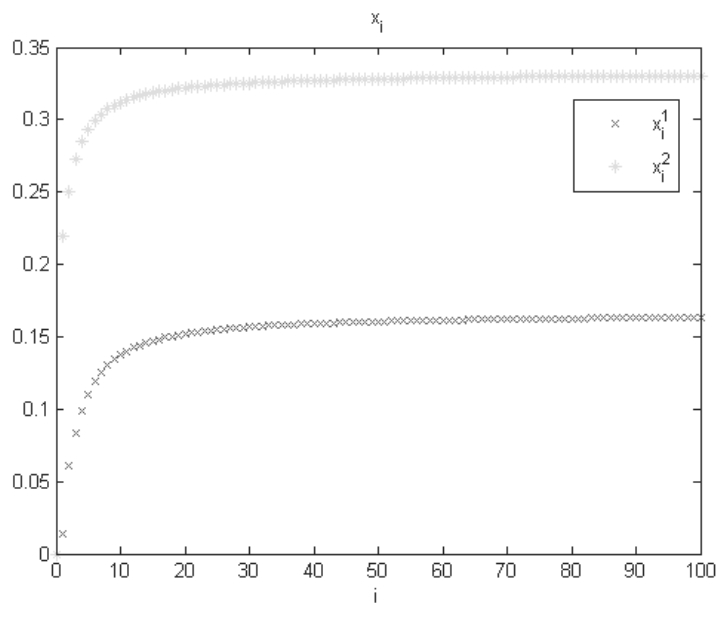

Fig. 2. Step response of the fractional discrete-time system (32).

Therefore, the fractional positive discrete-time system satisfies also the condition $\left|z_{k}\right|<1$ for $k=$ $1, \ldots, n$.

The discussion of stabilization by state feedbacks of positive systems presented by Kaczorek (2013) can be easily extended to fractional positive linear systems.

\section{Concluding remarks}

The approximation of fractional positive asymptotically stable continuous-time linear systems with the use of a linear Padé-type approximation by fractional positive asymptotically stable systems was addressed. It was shown that the approximation preserves the positivity and asymptotic stability of the systems (Theorem 13). The optimal choice of the coefficient $\beta$ so that the square of the difference between solutions of continuous-time and discrete-time systems be minimal was also discussed. The discussion was illustrated with a numerical example. The presented approach can be extended to fractional 2D linear systems (Kaczorek, 2011).

\section{Acknowledgment}

I wish to thank very much Dr. Krzysztof Rogowski for his valuable remarks and computation of Example 1. This work was supported by the National Science Centre in Poland under the grant no. N N514 638940.

\section{References}

Berman, A. and Plemmons, R.J. (1994). Nonnegative Matrices in the Mathematical Sciences, SIAM, Philadelphia, PA.

Busłowicz, M. (2008). Stability of linear continuous-time fractional order systems with delays of the retarded type, Bulletin of the Polish Academy of Sciences: Technical Sciences 56(4): 319-324.
Busłowicz, M. (2012). Stability analysis of continuous-time linear systems consisting of $n$ subsystems with different fractional orders, Bulletin of the Polish Academy of Sciences: Technical Sciences 60(2): 279-284.

Busłowicz, M. and Kaczorek, T. (2009). Simple conditions for practical stability of positive fractional discrete-time linear systems, International Journal of Applied Mathematics and Computer Science 19(2): 263-269, DOI: 10.2478/v10006-009-0022-6.

Farina, L. and Rinaldi, S. (2000). Positive Linear Systems: Theory and Applications, J. Wiley, New York, NY.

Gantmakher, F.R. (1959). Theory of Matrices, Chelsea Pub. Co., New York, NY.

Kaczorek, T. (2002). Positive $1 D$ and 2D Systems, Springer-Verlag, London.

Kaczorek, T. (1999). Relationship between the value of discretisation step and positivity and stabilization of linear dynamic systems, Proceedings of the Conference on Simulation, Designing and Control of Foundry Processes, Kraków, Poland, pp. 33-39.

Kaczorek, T. (1998). Vectors and Matrices in Automation and Electrotechnics, WNT, Warsaw, (in Polish).

Kaczorek, T. (2011). Selected Problems of Fractional System Theory, Springer-Verlag, Berlin.

Kaczorek, T. (2013). Approximation of positive stable continuous-time linear systems by positive stable discrete-time systems, Pomiary Automatyka Robotyka 59 (2): 359-364.

Kaczorek, T. (2011). Necessary and sufficient conditions of stability of fractional positive continuous-time linear systems, Acta Mechanica et Automatica 5(2): 52-54.

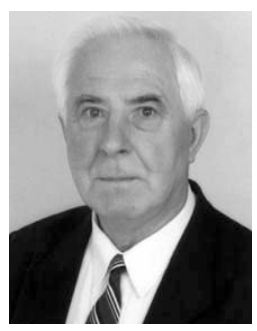

Tadeusz Kaczorek received the M.Sc., Ph.D and D.Sc. degrees in electrical engineering from the Warsaw University of Technology in 1956, 1962 and 1964, respectively. In the years 1968-69 he was the dean of the Electrical Engineering Faculty, and in the period of 1970-73 he was a deputy rector of the Warsaw University of Technology. In 1971 he became a professor and in 1974 a full professor at the same university. Since 2003 he has been a professor at Białystok Technical University. In 1986 he was elected a corresponding member and in 1996 a full member of the Polish Academy of Sciences. In the years 1988-1991 he was the director of the Research Centre of the Polish Academy of Sciences in Rome. In 2004 he was elected an honorary member of the Hungarian Academy of Sciences. He has been granted honorary doctorates by nine universities. His research interests cover systems theory, especially singular multidimensional systems, positive multidimensional systems, singular positive 1D and 2D systems, as well as positive fractional $1 \mathrm{D}$ and $2 \mathrm{D}$ systems. He initiated research in the field of singular 2D, positive $2 \mathrm{D}$ and positive fractional linear systems. He has published 24 books (six in English) and over 1000 scientific papers. He has also supervised $69 \mathrm{Ph}$.D. theses. He is the editor-in-chief of the Bulletin of the Polish Academy of Sciences: Technical Sciences and a member of editorial boards of ten international journals. 


\section{Appendix}

Lemma A1. If $s_{k}, k=1,2, \ldots, n$, are eigenvalues of the matrix $A_{c} \in M_{n}$, then the eigenvalues $z_{k}, k=$ $1,2, \ldots, n$, of the matrix

$$
A_{d}=\left[A_{c}+I_{n} \beta\right]\left[I_{n} \beta-A_{c}\right]^{-1}
$$

are given by

$$
z_{k}=\frac{s_{k}+\beta}{\beta-s_{k}} \quad \text { for } \quad k=1,2, \ldots, n .
$$

Proof. If $A_{c} \in M_{n}, \beta>0$ is chosen so that $\left[A_{c}+I_{n} \beta\right] \in$ $\mathbb{R}_{+}^{n \times n}$ and $\beta \neq s_{k}$, then the function

$$
f\left(s_{k}\right)=\frac{s_{k}+\beta}{\beta-s_{k}}
$$

is well defined on the spectrum $s_{k}, k=1,2, \ldots, n$, of the matrix $A_{c}$. In this case it is well known (Gantmakher, 1959; Kaczorek, 1998; 2013) that the equality (A2) holds.

Received: 7 March 2013

Revised: 12 June 2013 